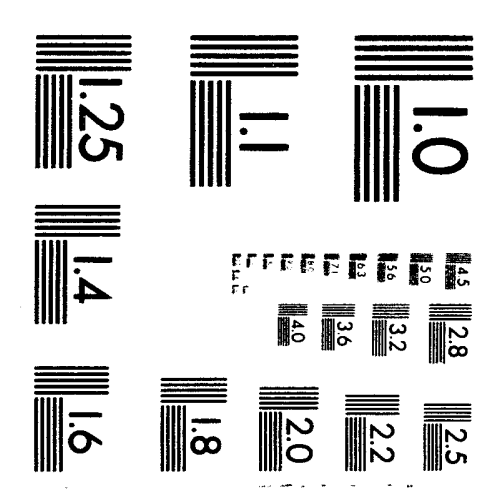



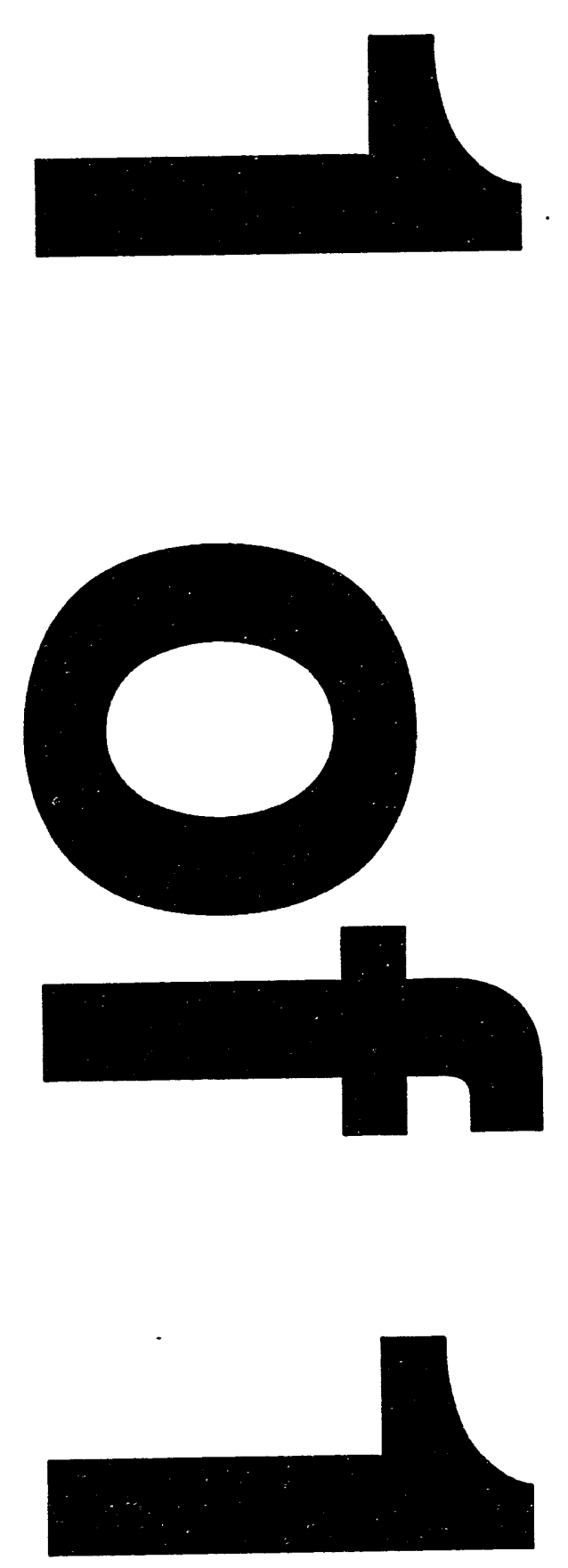


\section{NOTICE}

This report was prepared as an account of work sponsored by an agency of the United States Government. Neither the United States nor any agency thereof, nor any of their employees, makes any warranty, expressed or implied, or assumes any legal liability or responsibility for any third party's use or the results of such use of any information, apparatus, product or process disclosed in this report, or represents that its use by such third party would not infringe privately owned rights.

Printed in the United States of America

Available from

National Technical Information Service

U.S. Department of Commerce

5285 Port Royal Road

Springfield, VA 22161

NTIS Price codes

Printed copy: A02

Microfiche copy: A01 


\title{
Confinement Improvement with rf Poloidal Current Drive in the Reversed-Field Pinch
}

\author{
S. Hokin, J. Sarff, C. Sovinec \\ Department of Physics, University of Wisconsin, Madison, WI 53706 \\ E. Uchimoto
}

Department of Physics and Astronomy, University of Montana, Missoula, MT 59812

March 8, 1994

\begin{abstract}
External control of the current profile in a reversed-field pinch (RFP), by means such as rf poloidal current drive, may have beneficial effects well beyond the direct reduction of Ohmic input power due to auxiliary heating. Reduction of magnetic turbulence associated with the dynamo, which drives poloidal current in a conventional RFP, may allow operation at lower density and higher electron temperature, for which rf current drive becomes efficient and the RFP operates in a more favorable regime on the $n \tau$ vs. $T$ diagram. Projected parameters for RFX at $2 \mathrm{MA}$ are studied as a concrete example. If rf current drive allows RFX to operate with $\beta=10 \%$ (plasma energy/magnetic energy) at low density $\left(3 \times 10^{19} \mathrm{~m}^{-3}\right)$ with classical resistivity (ie. without dynamo-enhanced power input), $40 \mathrm{~ms}$ energy confinement times and $3 \mathrm{keV}$ temperatures will result, matching the performance of tokamaks of similar size.
\end{abstract}

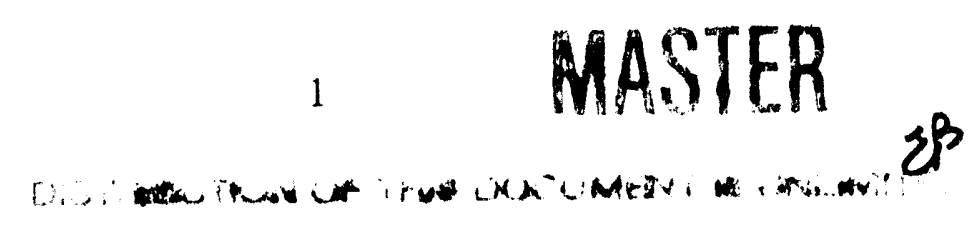




\section{INTRODUCTION}

The advantage of a conventional, Ohmic reversed-field pinch (RFP) as a fusion reactor lies in its confinement of a high-beta plasma with extremely high engineering beta (plasma pressure/magnetic field at coils) and no auxiliary heating. Its disadvantages include the empirical necessity of operating at high toroidal current and density $[1,2]$ to achieve good confinement and the detrimental transport effect of turbulence associated with the dynamo $[3,4,5]$, which drives poloidal current by means of the motion emf generated by coherent fluid velocity and magnetic field fluctuations. (Magnetic fluctuations have recently been measured to be a significant cause of energy transport from the RFP core [6].)

If one could make an RFP operate at constant beta (say, 10\%) as density is decreased without an associated increase of anomalous resistivity associated with the dynamo, several benefits would accumulate. First, the higher electron temperature results in sharply decreased Ohmic input power, ie. improved energy confinement. Second, although the density is reduced, the $n \tau$ product may remain constant or even increase while the temperature strongly increases, moving the RFP operating point towards the breakeven criterion on the $n \tau$ vs. $T$ diagram. Third, the higher electron temperature improves the efficiency of rf current drive.

Initial experimental results [7] and 3-D MHD simulations [8] indicate that energy confinement can be improved by external control of the current profile. In this article we examine the feasibility of using rf current drive to drive poloidal current, flatten the profile of $J_{\|} / B$, reducing the dynamo and its associated fluctuations, to maintain $10 \%$ beta in the RFX device [9] at $2 \mathrm{MA}$ and density as low as $3 \times 10^{19} \mathrm{~m}^{-3}$. We base our study on extrapolation of current performance of the two large RFPs, RFX [2] and MST [1], as well as results from smaller RFP devices. We focus on current drive using the slow wave with $\omega \sim 2 \omega_{L H}$, where $\omega_{L H}$ is the lower-hybrid frequency; a detailed study of propagation and current drive associated with fast magnetosonic waves has been reported in [10], and a preliminary study of slow wave current drive has been reported [11]. We 
find that RFX may produce improved confinement and temperatures at 2 MA under these circumstances. More detailed studies, including ray tracing and Fokker-Planck simulations, are underway and will be reported at a later time.

\section{IDEAL AND DEGRADED DHMIC RFP CONFINEMENT}

Many RFP groups have reported degradation of plasma beta with increasing $I / N$, where $I$ is the toroidal current, $N=\pi a^{2}\langle n\rangle$ and $a$ is the minor radius $[1,2,12,13] . I / N$ often is increased by increasing current at constant density; however, current and density have been independently varied in MST [1] with similar results. Beta degradation may be summarized with an approximate empirical relation

$$
\beta=\beta_{\max }\left(\frac{I / N}{2}\right)^{-0.6},
$$

where throughout this letter $I / N$ is in units of $10^{-14} \mathrm{Am} ; I / N=2$ is the high-density radiation limit observed on many RFPs.

Many RFPs have also reported an increase in the resistance anomaly with increasing $I / N$, as well as increased anomalous ion heating [14] associated with dynamo activity [15]. The decreased beta and increased Ohmic input power associated with the resistance anomaly lead to degradation of energy confinement with increasing $I / N$. Although this degradation is not understood, it may be associated with a change in the resistivity profile - at higher $I / N$, the edge resistivity may be higher relative to the center, enhancing the power dissipation associated with the dynamo, making it more inefficient. Small changes in the current density profile may affect tearing mode activity; fluctuation levels have been reported to increase with $I / N[16]$.

As a result, most RFP fusion reactor studies choose $I / N=2$ as an operating point [17]. Operation near the density limit requires very high current (18 MA in the case of the TITAN study) to reach fusion conditions, since

$$
T=\frac{3}{2} \beta \frac{I}{N} I
$$


where $T$ is in $\mathrm{keV}$ and $I$ is in MA. There is a great advantage to operating an RFP at higher $I / N$ (lower density, higher temperature) if beta can be maintained constant.

To begin, we wish to analyze a simple 'ideal' Ohmic RFP, in which there is no anomalous resistivity and beta is constant, independent of density. This is an unattainable ideal, since, for example, the Ohmic toroidal electric field cannot drive the current outside the reversal radius, where it opposes the local current density. A real steady-state Ohmic RFP must have a dynamo to drive part of the poloidal current, with an associated resistance anomaly. Without anomalous resistivy, there will be no anomalous ion heating; this is of no consequence, as RFX at $2 \mathrm{MA}$ (and a reactor) has collisionally coupled electrons and ions. We therefore assume $T_{i}=T_{e}$ in our estimate of energy confinement time, and calculate the ion-electron equilibration rate $\tau_{e q}^{i / e}$ as a check.

The RFP has a large enhancement of Ohmic input power over a tokamak of the same temperature and toroidal current due to the large poloidal current, summarized by the factor $g$ in the expression

$$
P_{S p}=g \frac{2 R}{a^{2}} \eta I^{2},
$$

where $\eta \propto Z_{e f f} T_{e}^{-3 / 2}$ is the Spitzer resistivity, assumed here to be independent of radius, and

$$
g \equiv \frac{a^{2}}{2} \frac{\int J^{2} r d r}{\left(\int J_{\phi}^{2} r d r\right)^{2}} \simeq 5 .
$$

The advantage of operation at higher $I / N$ and $T$ is then evident from the expression for the Spitzer energy confinement time:

$$
\tau_{E}^{S p}=127 \frac{a^{2}}{g Z_{e f f} \ln \Lambda} \frac{T^{5 / 2}}{I(I / N)},
$$

where $\tau_{E}^{S_{p}}$ is in s, $a$ is in $\mathrm{m}, T$ is in $\mathrm{keV}, I$ is in MA and $I / N$ is in $10^{-14} \mathrm{Am}$. Since $T \propto I / N$ for constant beta and current, $\tau_{E}$ scales as $(I / N)^{3 / 2}$.

We choose $\beta=\beta_{\max }=10 \%$, a value already achieved in many RFPs, and $Z_{\text {eff }}=2$. The resulting performance of RFX at $2 \mathrm{MA}$ in a density scan spanning $2<I / N<10$ is shown in Table I. At $I / N=10$ corresponding to $\langle n\rangle=3 \times 10^{19} \mathrm{~m}^{-3}$, our ideal RFX would achieve over $100 \mathrm{~ms}$ energy confinement time and $3 \mathrm{keV}$ temperature. 


\begin{tabular}{rr|rrrrr|rrrrrr} 
& & \multicolumn{5}{|c|}{$\beta=0.1$} & \multicolumn{5}{|c}{$\beta=0.1(I / N) / 2$} \\
\hline$I / N$ & $n$ & $T_{e}$ & $\tau_{e q}^{i / e}$ & $P_{S_{p}}$ & $\tau_{E}$ & $n \tau_{E}$ & $T_{e}$ & $\tau_{e q}^{i / e}$ & $P_{S_{p}}$ & $P_{\text {in }}$ & $\tau_{E}$ & $n \tau_{E}$ \\
\hline 2 & 15.6 & 0.60 & 1 & 39 & 9 & 0.14 & 0.60 & 1 & 39 & 40 & 8.9 & 0.134 \\
4 & 7.8 & 1.20 & 6 & 14 & 27 & 0.21 & 0.79 & 3 & 26 & 29 & 8.4 & 0.066 \\
6 & 5.2 & 1.80 & 15 & 8 & 49 & 0.26 & 0.94 & 6 & 20 & 25 & 7.7 & 0.040 \\
8 & 3.8 & 2.40 & 32 & 5 & 74 & 0.28 & 1.06 & 10 & 17 & 24 & 6.6 & 0.025 \\
10 & 3.1 & 3.00 & 55 & 4 & 105 & 0.33 & 1.14 & 13 & 15 & 25 & 5.6 & 0.017
\end{tabular}

Table I: Projected performance of an RFX 2 MA density scan with $Z_{\text {eff }}=2$, for the cases of constant beta without anomalous resistance and beta degradation with anomalous resistance. $I / N$ is in $10^{14} \mathrm{Am}, n$ is in $10^{19} \mathrm{~m}^{-3}, T$ is in $\mathrm{keV}, \tau$ is in $\mathrm{ms}, P$ is in $\mathrm{MW}$, and $n \tau$ is in $10^{19} \mathrm{~m}^{-3}$-s.

For comparison, we estimate the 'degraded' RFX energy confinement expected from extrapolation of existing data, using $Z_{\text {eff }}=2$ and beta degradation from Eq. 1 with $\beta_{\max }=10 \%$, adding the additional effect of anomalous resistance. In order to measure the resistance anomaly, which we quantify using the parameter

$$
\gamma \equiv \frac{P_{\text {in }}-P_{S p}}{P_{S p}}
$$

one must measure $Z_{\text {eff }}, T_{e}$ and the total input power $P_{\text {in }}$. The measurement of $Z_{\text {eff }}$ requires comprehensive spectroscopy, which has been lacking in many RFP experiments. Data reported for Extrap-T1 [16] $\left(Z_{\text {eff }} \simeq 2\right.$ for all conditions) in the range $7<I / N<15$ indicate that $\gamma \propto\left(T_{e} / n_{e}\right)^{1.5}$, or, choosing $I / N$ as the independent parameter with some loss of fit quality,

$$
\gamma \simeq 7 \times 10^{-3}(I / N)^{2} .
$$

The anomalous input power is comparable to the Spitzer power at $I / N=10$. Early data from RFX with $I<0.7 \mathrm{MA}[18]$ indicate that $\gamma$ is small with $Z_{\text {eff }} \simeq 4$ over the range $2<I / N<5$, in which Eq. 7 predicts $0.03<\gamma<0.18$.

Our estimate of degraded confinement for RFX at $2 \mathrm{MA}$ is also presented in Table I. The degradation of energy confinement with increasing $I / N$, observed on many RFPs 
including RFX [2], is reproduced by our beta and anomalous power scaling relations (Eqs. 1 and 7). The $n \tau_{E} T$ product is reduced by a factor of 50 at $I / N=10$ from its 'ideal' value for $\beta=10 \%$ and classical input power.

The degradation of beta and increase of anomalous resistance with increasing $I / N$ are clear impediments to achieving a compact RFP fusion reactor. They are related the beta degradation is a result of degraded energy confinement, likely produced by the increased magnetic fluctuation activity which heats ions [15] and results in anomalous input power. If one can reduce the magnetic fluctuations at high $I / N$, by flattening the current profile, one may be able to achieve remarkably improved performance. In fact, transient inductive current profile control has been shown to double the energy confinement time in MST [7]. In the next section, we discuss lower hybrid current drive as a candidate possibility for current profile control in RFX.

\section{LOWER HYBRID CURRENT DRIVE}

Lower hybrid current drive (LHCD, $\omega \sim 2 \omega_{L H}$ ) has proven to be a successful method of external current drive and profile control in tokamaks. It, or any other external current drive technique, remains to be tested in the RFP. Fast magnetosonic waves are predicted to drive current efficiently [10] for parameters similar to those studied here; the slow (LH) wave has the advantage of higher frequency ( $1 \mathrm{GHz}$ for RFX) with an associated simplification of antenna and rf technology. For this reason, we make simple analytical estimates of the requirements on a LH system for profile control in RFX.

One does not need to drive all of the poloidal current in the RFP to replace the dynamo. Part of the applied toroidal electric field $E_{\phi}$ results in poloidal current due to the helical magnetic geometry.

One can determine the poloidal current required of the current drive scheme using parallel Ohm's law with an ad hoc force term $\mathcal{E}$ representing the dynamo or current drive:

$$
\mathbf{E}_{\|}+\mathcal{E}_{\|}=\eta \mathbf{J}
$$


For example, the MHD dynamo is $\mathcal{E}=\langle\tilde{\mathbf{V}} \times \tilde{\mathbf{B}}\rangle$, and opposes $\mathbf{E}$ or axis, resulting in the resistance anomaly. Electric field and resistivity are related for ali ideal RFP (with no resistance anomaly) since $E_{\phi}=\eta_{0} J_{\phi}(0)$. The applied toroidal voltage drives the current density fraction $\mathbf{E}_{\|} / \eta$ while the dynamo or current drive drives the remainder. Taking the poloidal component, setting $E_{\phi}=\eta_{0} J_{\phi}(0)$ and integrating, one gets the fraction of poloidal current driven by the toroidal circuit:

$$
\frac{I_{\theta}^{E}}{I_{\theta}}=\frac{\int d r \eta J_{\phi}(0) B_{\theta} B_{\phi} / B^{2}}{\eta_{0} \int d r J_{\theta}}
$$

This ratio is sensitive to the resistivity profile. For $\eta=$ constant, this ratio is 0.76 for the model equilibrium used in our study; for $\eta / \eta_{0}=1+24(r / a)^{6}$, a more realistic profile, this ratio is 0.57 . (A great benefit arises from flattening the resistivity profile, which is not addressed in this article.) The poloidal current drive system must therefore drive $30-50 \%$ of the poloidal current. At $2 \mathrm{MA}, I_{\theta} \simeq 3 I R / a=30 \mathrm{MA}$, implying that $9-15 \mathrm{MA}$ of poloidal current drive is required in RFX, in addition to any extra current deposited for profile modification.

In order to suppress tearing mode fluctuations, the profile of $\lambda \equiv \mu_{0} a J / B$ must be flattened. (We use a force-free equilibrium model, so $J_{\|}=J$.) The amount of required flattening may not be large, given that the dynamo fluctuations may maintain the equilibrium near marginal stability. We have analyzed the stability of two $\lambda$ profiles, similar to those shown in Fig. 1, with the 3-D MHD code DEBS [19] for $R / a=3$ and Lundquist number $S=10^{4}$. The undriven profile corresponds to $F=-0.15, \Theta=1.47$, an actual operating point of RFX at $500 \mathrm{kA}$. The current-driven profile has an extra portion with th- shape of a Gaussian with FWHM $0.2 \mathrm{a}$ and peaked at $r / a=0.8$, totalling 1.2 MA additional poloidal current. The current-driven profile results in good flux surfaces out to $r / a=0.6$ while the undriven profile has no flux surfaces. The driven profile has over a factor of 10 reduction of tearing mode amplitudes.

In order to estimate current drive power requirements, we assume that the system must drive $40 \%$ of the poloidal current (midway between our flat and hollow resistivity profile estimates), or $12 \mathrm{MA}$, to replace the dynamo plus an additional localized $1.2 \mathrm{MA}$ 


\begin{tabular}{rr|rrrrrr|rrrrrr} 
& & \multicolumn{7}{|c|}{$\beta=0.1$} & \multicolumn{5}{|c}{$\beta=0.1(I / N) / 2$} \\
\hline$I / N$ & $n$ & $T_{e}$ & $w$ & $\tilde{\eta}$ & $\eta$ & $P_{c d}$ & $\tau_{E}$ & $T_{e}$ & $w$ & $\tilde{\eta}$ & $\eta$ & $P_{c d}$ & $\tau_{E}$ \\
\hline 2 & 15.6 & 0.60 & 2.5 & 7 & 0.1 & 415 & 1 & 0.60 & 2.5 & 7 & 0.07 & 415 & 0.8 \\
4 & 7.8 & 1.20 & 3.5 & 10 & 0.4 & 76 & 4 & 0.79 & 2.8 & 7 & 0.18 & 165 & 1.3 \\
6 & 5.2 & 1.80 & 4.3 & 13 & 1.2 & 26 & 11 & 0.94 & 3.1 & 8 & 0.37 & 81 & 1.9 \\
8 & 3.8 & 2.40 & 5.0 & 16 & 2.6 & 12 & 22 & 1.06 & 3.3 & 9 & 0.64 & 47 & 2.5 \\
10 & 3.1 & 3.00 & 5.5 & 19 & 4.7 & 6 & 37 & 1.14 & 3.4 & 10 & 0.94 & 32 & 3.0
\end{tabular}

Table II: Projected current drive power requirements for the RFX 2 MA density scan of Table I with $I_{c d}=13 \mathrm{MA}$. Units are the same as Table $\mathrm{I} ; \eta$ is in $\Lambda \mathrm{m} / \mathrm{W}$.

to provide the required flattening. We assume the current drive to be peaked at $r / a=0.8$, with diffusion providing inward transport of the driven current. Our main goal here is power requirements; details of current-driven profiles in the presence of transport must be worked out with ray tracing and transport calculations.

At $2 \mathrm{MA}, B=8.75 \mathrm{kG}$ at the RFX plasma edge, suggesting a frequency choice of 1 $\mathrm{GHz}$. In order to have good accessibility to $r / a=0.8$ and significant electron Landau damping, a good choice for the wave launch is $n_{\|}=5$, which will geometrically transform to $n_{\|} \sim 7$ at $r / a=0.8$. The LHCD efficiency $\eta(\mathrm{Am} / \mathrm{W})$ has been calculated [20]:

$$
\eta=3.84 \times 10^{19} \frac{T_{e}}{n_{e} \ln \Lambda} \tilde{\eta}(w),
$$

where $w=\omega / k_{\|} v_{t e}$ and the normalized efficiency $\tilde{\eta}$ is plotted in [20]. Since $T_{e} / n_{e}$ increases with $I / N$, it is evident that the LHCD efficiency increases with $I / N$. The required LHCD power is $P_{c d}=2 \pi r I_{c d} / \eta$ with $2 \pi r=2.3 \mathrm{~m}$ for absorption centered at $r / a=0.8$.

The power requirements are displayed in Table II, corresponding to the parameters of Table I. A modified energy confinement time is shown, which includes both the rf input $P_{c d}$ and Ohmic power $P_{S p}$. Since $P_{c d}>P_{S_{p}}$ in all cases, one might expect that additional heating will result from the $\mathrm{rf}$, which we do not include, improving the confinement time. The projected parameters at high density are clearly prohibitive for rf current drive due 
to low efficiency. However, the case with no anomalous power input at $I / N=10$ has $\tau_{E}=37 \mathrm{~ms}$, a $65 \%$ reduction from the Ohmic-only ideal case due to the added $6 \mathrm{MW} \mathrm{rf}$ power, but a $660 \%$ improvement over the degraded confinement of 5.6 ms projected with Eqs. 1 and 7 for beta degradation and anomalous resistance. Our results are summarized on the Lawson diagram of Fig. 2.

Even if this scenario proves correct, there remains a problem with the initial (degraded) target plasma having too low an electron temperature for efficient current drive; the discharge must 'bootstrap' to good confinement once the rf power has been added. A high-power short-pulse startup section might be required for the initial profile adjustment. This section may have to deliver $25 \mathrm{MW}$ or more power for a brief time, to be followed by a longer pulse section at 6.4 MW. An alternative may be the application of a transient poloidal voltage before application of $\mathrm{rf}$. Initial profile control experiments using this technique on MST [7] indicate that the time scale for profile modification is very short, less than $1 \mathrm{~ms}$ for $T_{e}=200 \mathrm{eV}$ in a plasma of dimensions similar to RFX.

\section{CONCLUSIONS}

We have reported an initial study of the benefits of poloidal current drive in the RFP, with the goal of estimating the improvement of confinement which might result if current drive allows the RFP to operate at low density $(I / N=10)$ and high beta $(10 \%)$ in

the absence of anomalous resistance. Although our results are speculative and modeldependent, there is a clear benefit to optimized low-density operation - both the classical confinement time and of current drive efficiency are greatly improved.

We find, under our model assumptions, that the energy confinement time may be improved by over a factor of 6 if the resistance anomaly and beta degradation are eliminated by application of lower hybrid current drive in RFX at 2 MA. We propose a system at $f=1 \mathrm{GHz}$ with a launch structure designed for $n_{\|}=5$. Detailed ray tracing and kinetic calculations will be reported in the future.

Even if RFX performs much better than our 'degraded' confinement scaling predictions, there will remain a benefit in operation with reduced fluctuations associated with 
current profile control. A near-term goal of RFX operation should be to investigate our degradation model (Eqs. 1 and 7) by performing density scans at various current levels. There is clearly a long-term advantage to developing optimized operation of the RFP at moderate to low density.

\section{Acknowledgements}

This work was supported by the University of Wisconsin, the University of Montana and the U.S. Department of Energy. 


\section{References}

[1] S.A. Hokin and et al, in Proceedings of the 20th European Conf. on Contr. Fusion and Plasma Physics, Lisbon, edited by J.A. Costa Cabral, M.E. Manso, F.M. Derra, and F.C. Schuller (EPS, Centro de Fusão Nuclear, Instituto Superior Tecnico, Lisbon, 1993), Vol. 17C Part II, p. 475.

[2] V. Antoni and et al., Plasma Phys. Controlled Fusion 35, B333 (1993).

[3] K. Kusano and T. Sato, Nucl. Fusion 27, 821 (1987).

[4] R.A. Nebel, E.J. Caramana, and D.D. Schnack, Phys. Fluids B 1, 1671 (1989).

[5] Y.L. Ho and G.G. Craddock, Phys. Fluids B 3, 721 (1991).

[6] G. Fiksel, S. Prager, W. Shen, and M. Stoneking, Phys. Rev. Lett. 72, 1028 (1994).

[7] J. Sarff, S. Hokin, S. Prager, and H. Ji, submitted to Phys. Rev. Lett. (1994).

[8] Y.L. Ho, Nucl. Fusion 31, 341 (1991).

[9] G. Malesani, in Proceedings of the International School of Plasma Physics Workshop on Physics of Mirrors, Reversed Field Pinches and Compact Tori, Varenna, Italy, 1986, edited by S. Ortolani and E. Sindoni (Società Italiana di Fisica, Bologna, 1987), p. 359.

[10] H. Ishii, Y. Kondoh, T. Shimada, Y. Hirano, S. Shiina, and K. Saito, in Proceedings of the 20th European Conf. on Contr. Fusion and Plasma Physics, Lisbon, edited by J.A. Costa Cabral, M.E. Manso, F.M. Derra, and F.C. Schuller (EPS, Centro de Fusão Nuclear, Instituto Superior Tecnico, Lisbon, 1993), Vol. 17C Part II, p. 495.

[11] E. Uchimoto, M. Cekic, C. Litwin, S. Prager, J. Sarf, and C. Sovinec, in Bull. Am. Phys. Soc., (AIP, 1993) Vol. 38, p. 1979.

[12] B. Alper and et al., in Plasma Physics and Controlled Nuclear Fusion Research, 1986, (International Atomic Energy Agency, Vienna, 1986), Vol. 2, p. 399. 
[13] T. Shimada and et al., in Plasma Physics and Controlled Nuclear Fusion Research, 1988, (International Atomic Energy Agency, Vienna, 1989), Vol. 2, p. 457.

[14] S. Hokin and et al., Journal of Fusion Energy 12, 281 (1993).

[15] E. Scime, M. Cekic, D.J. Den Hartog, S. Hokin, D. Holly, and C. Watts, Phys. Fluids B 4, 4062 (1992).

[16] J.H. Brzozowski, J.R. Drake, S. Mazur, P. Nordlund, and K.-D. Zastrow, in Proceedings of the 20th European Conf. on Contr. Fusion and Plasma Physics, Lisbon, edited by J.A. Costa Cabral, M.E. Manso, F.M. Derra, and F.C. Schuller (EPS, Centro de Fusão Nuclear, Instituto Superior Tecnico, Lisbon, 1993), Vol. 17C Part II, p. 467.

[17] F. Najmabadi et al., UCLA report UCLA-PPG-1200 UCLA (1990).

[18] L. Carraro, M.E. Puiatti, P. Scarin, and M. Valisa, in Proceedings of the 20th European Conf. on Contr. Fusion and Plasma Physics, Lisbon, edited by J.A. Costa Cabral, M.E. Manso, F.M. Derra, and F.C. Schuller (EPS, Centro de Fusão Nuclear, Instituto Superior Tecnico, Lisbon, 1993), Vol. 17C Part II, p. 451.

[19] D.D. Schnack, D.C. Barnes, Z. Mikic, D.S. Harned, and E.J. Caramana, J. Comput. Phys. 70, 330 (1987).

[20] D.A. Ehst and C.F.F. Karney, Nucl. Fusion 31, 1933 (1991).

[21] V. Antoni, D. Merlin, S. Ortolani, and R. Paccagnella, Nucl. Fusion 26, 1711 (1986). 


\section{Figure Captions}

Figure 1 Profile of $\lambda=\mu_{0} a J / B$ without and with poloidal current drive providing an additional 1.2 MA localized current. The added current is represented by a Gaussian centered at $r / a=0.8$ with FWHM equal to $0.2 a$. The undriven profile is given by the 'alpha model' [21] with $\alpha=5.0, \Theta_{0}=1.514$ for which $F=-0.15, \Theta=1.47$; the driven profile has $\Theta=1.30$ and $F=-0.13$ with $B_{\phi}(a)$ held constant for the two cases. The current driven profile has good flux surfaces out to $r / a=0.6$.

Figure 2 Lawson diagram, indicating the projected performance of RFX at $2 \mathrm{MA}$ for $(+)$ ideal confinement, Ohmic drive only; $(*)$ degraded confinement, Ohmic drive only; ( $)$ ideal confinement with Ohmic and LHCD. 


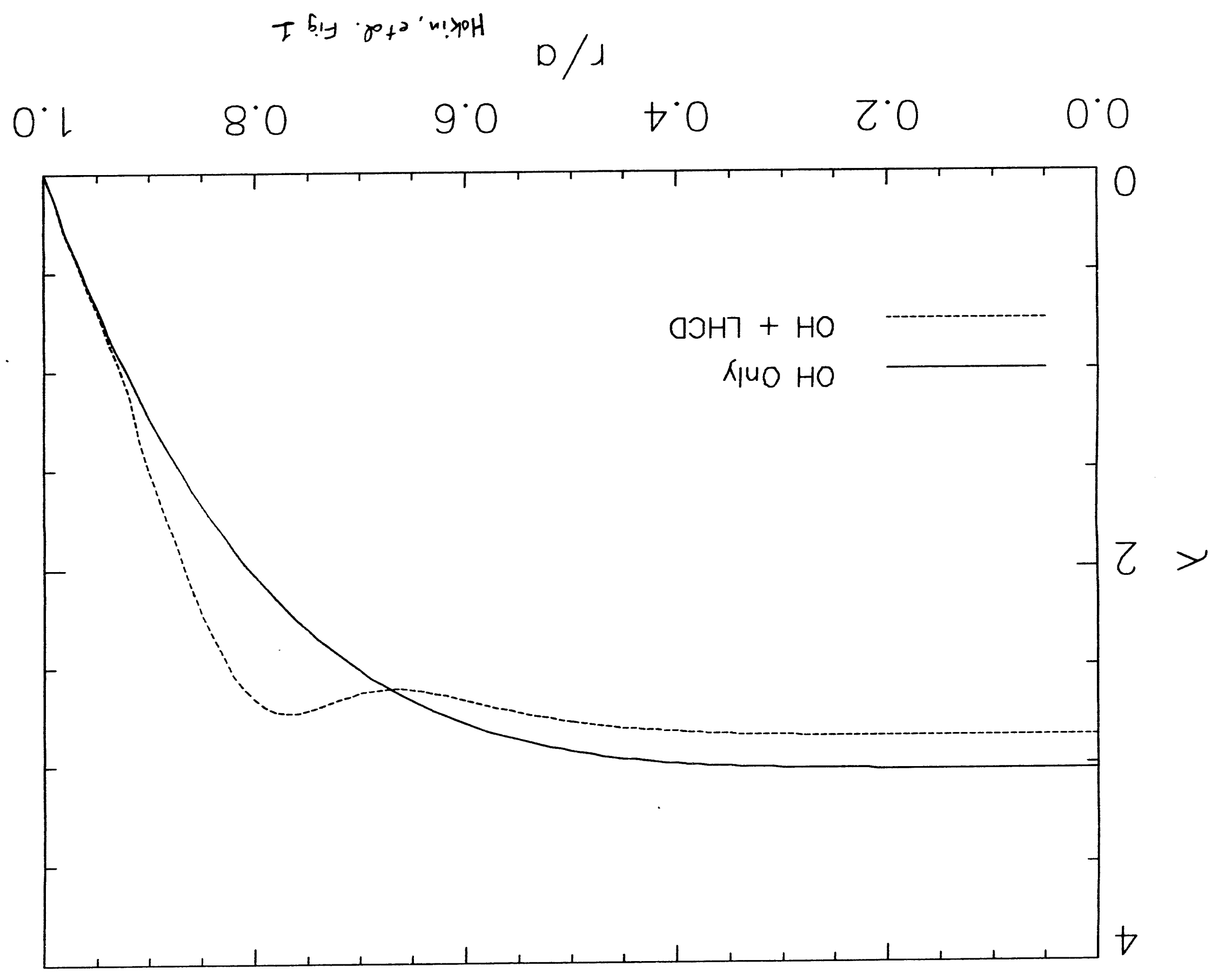




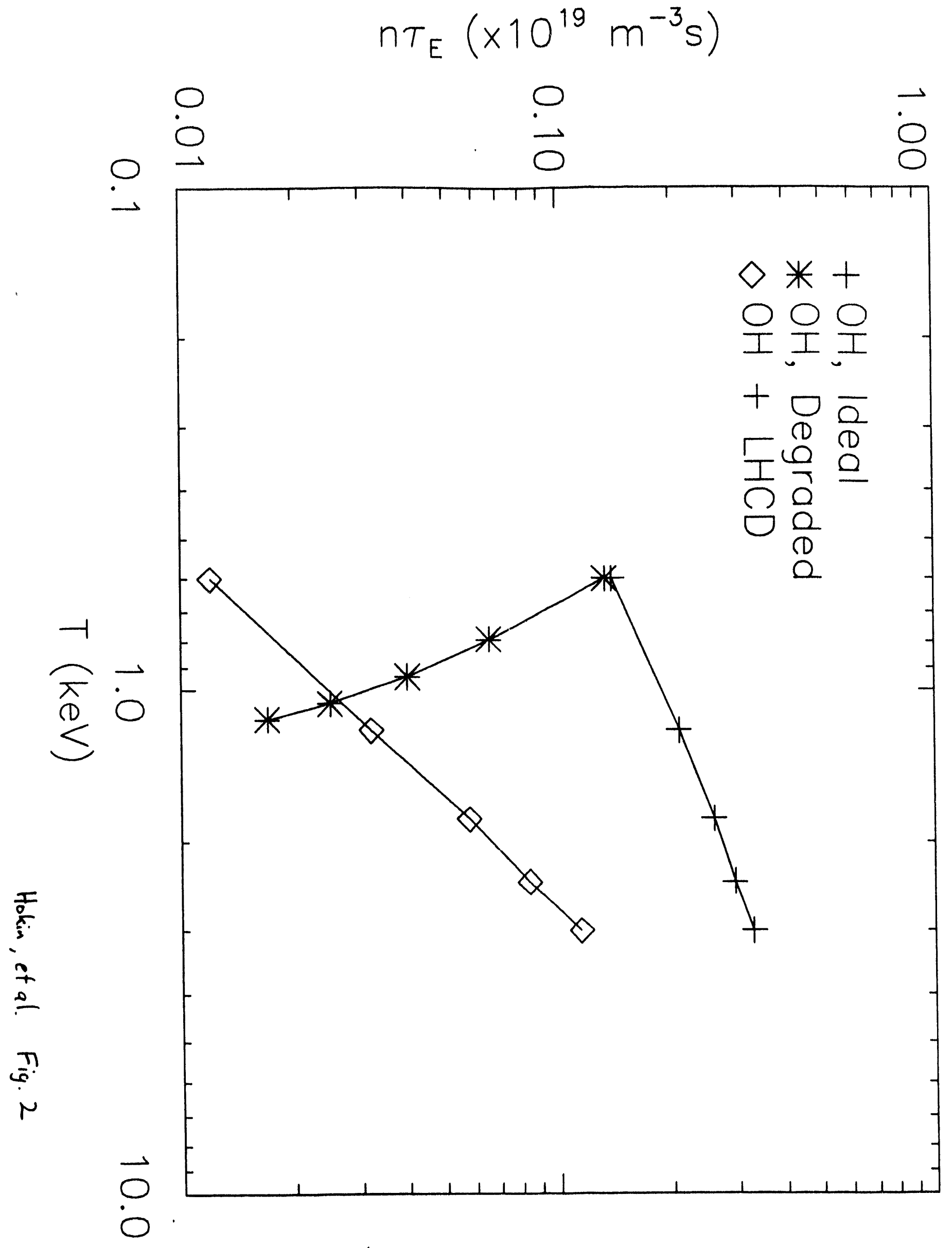


S.N. Rasband, Brigham Young University

T. Dolan, EG\&G Idaho, Inc.

R.A. Moyer, General Atomics

J.B. Taylor, Institute for Fusion Studies, The University of Texas at Austin

E. Uchimoto, University of Montana

F.W. Perkins, PPPL

O. Ishihara, Texas Technical University

M.A. Abdou, University of California, Los Angeles

R.W. Conn, University of California, Los Angeles

P.E. Vandenplas, Association Euratom-Etat Belge, Belgium

Centro Brasileiro de Pesquisas Firicas, Brazil

P. Sakanaka, Institute de Fisica-Unicamp, Brazil

Mme. Monique Bex, GANIL, France

J. Radet, CEN/CADARACHE, France

University of Ioannina, Greece

S. Ortolani, Istituto Gas Ionizzati, EURATON-ENEA-CNR Association, Italy

R. Andreani, Associazione EURATOM-ENEA sulla Fusione, Italy

Plasma section, Energy Fundamentals Division Electrotechnical Laboratory, Japan

Y. Kondoh, Gunma University, Kiryu, Gunma, Japan

H. Toyama, University of Tokyo, Japan

Z. Ypsjoda. University of Tokyo, Japan

FOM-Instituut voor Plasmafysica "Rijnhuizen," The Netherlands

Z. Ning, Academia Sinica, Peoples Republic of China

P. Yang, Shandong University, Peoples Republic of China

S. Zhu, University of Science \& Technology of China, People's Republic of China

I.N. Bogatu, Institute of Atomic Physics, Romania

M.J. Alport, University of Natal, Durban, South Africa

R. Storer, The Flinders University of South Australia, South Australia

B. Lehnert, Royal Institute of Technology, Sweden

Librarian, CRPP, Ecole Polytechnique Federale de Lausanne, Switzerland

B. Alper, Culham Laboratory, UK

A. Newton, UK

2 for Chicago Operations Office

5 for individuals in Washington Offices

INTERNAL DISTRIBUTION IN ADDITION TO UC-20

80 for local group and file 

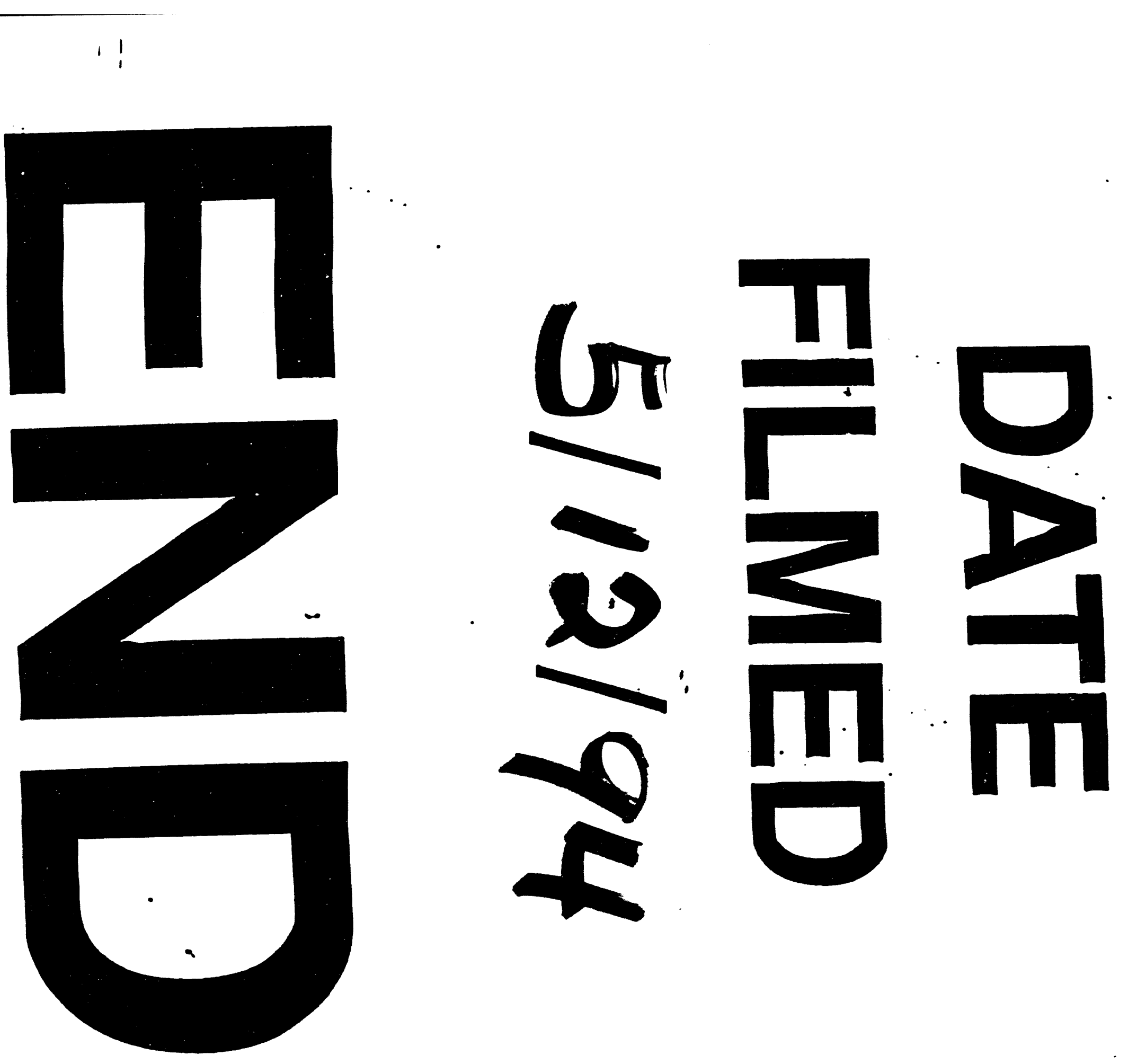


$$
2
$$

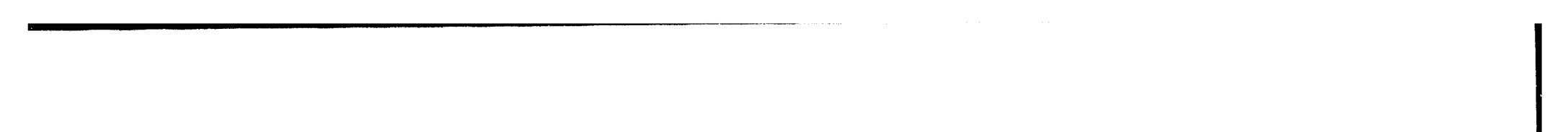

